\title{
The Yin and Yang of RNA surveillance in B lymphocytes and antibody-secreting plasma cells
}

\author{
Jean-Marie Lambert, Nivine Srour ${ }^{\dagger} \mathcal{E}^{\mathrm{E}}$ Laurent Delpy ${ }^{*}$ \\ UMR CNRS 7276 - INSERM 1268 - Université de Limoges, Centre de Biologie et de Recherche en Santé, 2 rue du Dr Marcland, Limoges \\ F-87025, France
}

The random V(D)J recombination process ensures the diversity of the primary immunoglobulin (lg) repertoire. In two thirds of cases, imprecise recombination between variable $(\mathrm{V})$, diversity (D), and joining (J) segments induces a frameshift in the open reading frame that leads to the appearance of premature termination codons (PTCs). Thus, many B lineage cells harbour biallelic V(D)J-rearrangements of Ig heavy or light chain genes, with a productively-recombined allele encoding the functional Ig chain and a nonproductive allele potentially encoding truncated Ig polypeptides. Since the pattern of Ig gene expression is mostly biallelic, transcription initiated from nonproductive Ig alleles generates considerable amounts of primary transcripts with out-of-frame V(D)J junctions. How RNA surveillance pathways cooperate to control the noise from nonproductive Ig genes will be discussed in this review, focusing on the benefits of nonsense- mediated mRNA decay (NMD) activation during B-cell development and detrimental effects of nonsense-associated altered splicing (NAS) in terminally differentiated plasma cells. [BMB Reports 2019; 52(12): 671-678]

\section{INTRODUCTION}

To ensure the fidelity of gene expression and prevent translation of truncated proteins, many cellular RNA surveillance pathways have been developed to distinguish between normal transcripts and those harboring premature termination codons (PTCs). RNA surveillance mechanisms

*Corresponding author. Tel: +33-519-564-214; Fax: +33-555-435897; E-mail: laurent.delpy@unilim.fr.

${ }^{\dagger}$ Present address: Lady Davis Institute for Medical Research, McGill University, 3755 Cote Ste-Catherine Road, Montreal, Quebec H3T 1E2, Canada.

https://doi.org/10.5483/BMBRep.2019.52.12.232

Received 23 September 2019

Keywords: Immunoglobulin, Nonsense-associated altered splicing (NAS), Nonsense-mediated mRNA decay (NMD), Plasma cells, RNA surveillance have been extensively studied in lymphoid cells that naturally acquire PTCs at high frequencies during the error-prone V(D)J recombination process (1). Through the analysis of nonproductive (PTC+) immunoglobulin (Ig) and T-cell receptor (TCR) transcripts, it has been demonstrated that transcriptional silencing, splicing inhibition or suppression (SOS), alternative splicing, and mRNA degradation cooperate with each other to limit the amount of potentially deleterious truncated proteins (2-12).

The nonsense-mediated mRNA decay (NMD) pathway is very active in lymphoid cells. It ensures rapid degradation of PTC-containing mRNAs $(1,9,13)$. The nearly complete absence of PTC + Ig or TCR mRNAs due to their strong NMD degradation can lead to the assumption that transcription of PTC + alleles is safe. Accordingly, nonproductive alleles are often considered as passengers in comparison with the driving effects of productively-rearranged Ig or TCR alleles during lymphoid development. Although the benefits of NMD activation are clearly established in lymphoid cells $(14,15)$, the impact of nonsense-associated altered splicing (NAS) with regard to the production of truncated Ig and TCR polypeptides remains unclear. The objective of this review is to summarize our current knowledge on the opposite effects of NMD and NAS during B cell development. This paradox will be discussed in line with our recent findings showing that NAS events can lead to the production of truncated Igא polypeptides that can blunt plasma cell (PC) differentiation (16). Challenging the classical antigen-driven PC differentiation model, this new PC checkpoint suggests that the expression of PTC + Ig genes can sometimes be disabling.

\section{THE GENERATION OF ANTIBODY REPERTOIRE: A RISKY DIVERSITY}

The error-prone $\mathrm{V}(\mathrm{D}) \mathrm{J}$ recombination process

Ig genes are good candidates to study nonsense RNA surveillance because the generation of primary Ig repertoire in early B-cell development and the process of somatic hypermutations (SHM) in germinal center B cells frequently generate PTCs (1). The $\mathrm{V}(\mathrm{D})$ ) recombination process of $\mathrm{Ig}$ genes takes place in the bone marrow and assembles the variable region from germline variable $(\mathrm{V})$, diversity $(\mathrm{D})$, and joining $(\mathrm{J})$ gene segments. Control of $\mathrm{V}(\mathrm{D}) \mathrm{J}$ recombination 
occurs at several levels, including cell-type specificity, intraand inter-locus sequential rearrangements, and allelic exclusion (17). Although DNA rearrangements in the Ig heavy $(\operatorname{lgH})$ and light (i.e. $\lg \kappa$ and $\lg \lambda$ ) loci occur in a precise order, the selection of gene segments within each locus is random. It allows for combinatorial diversity. The mechanism used by lymphoid cells to successfully rearrange their antigen (Ag) receptor genes requires the use of recombinase enzymes RAG1 \& RAG2 that are only active in lymphocytes $(18,19)$. Recombinases act at early stages of lymphoid cell development in order to bring two segments into close proximity, forming a loop of intervening DNA which can then be excised. The ends of these segments are annealed to form a newly rearranged DNA sequence. To increase diversity, joining of $\mathrm{V}, \mathrm{D}$, and J segments is imprecise with nucleotide deletions or insertions. Non-template $(\mathrm{N})$ nucleotide additions are introduced by terminal deoxynucleotidyl transferase (TdT). Palindromic (P) insertions occur after asymmetric hairpin opening. Random $\mathrm{N}$-additions cannot be attributed to any other genomic sequences. They rarely exceed a dozen nucleotides $(20,21)$. They are polymerized by TdT which is the third lymphoidspecific protein involved in $\mathrm{V}(\mathrm{D})$ ) recombination besides RAG1 and RAG2 (22-25). P insertions rarely exceed two nucleotides and form a palindrome with respect to the sequence at the end of the coding strand (26-28). Although nucleotide deletions and insertions greatly enlarge the diversity of the Ig repertoire, only one third of all V(D)J junctions are in-frame, while the other two thirds are out-of-frame due to frameshift mutations that create PTCs.

\section{Frequency of PTC-containing Ig genes in B-lineage cells}

Clonal selection implies that each $\mathrm{B}$ cell clone expresses a unique receptor. Hence, one of the two inherited Ig alleles is functionally rearranged. This allelic exclusion associates asynchronous $\mathrm{V}(\mathrm{D})$ ) recombination events at Ig loci with receptor-mediated inhibitory feedback control (29). At the pro-B cell stage, VDJ recombination is initiated by biallelic $\mathrm{D}$ to J rearrangements at IgH loci, followed by a monoallelic $\mathrm{V}$ to DJ recombination. A productive VDJ junction encodes the variable $(\mathrm{V})$ region of the $\mu$ heavy chain that can associate with the surrogate light chain to form pre-BCR (pre-B cell receptor). Regulatory mechanisms mediated by pre-BCR signaling prevent further $\mathrm{V}$ to $\mathrm{DJ}$ rearrangements on the second $\mathrm{IgH}$ allele and initiates VJ recombination at Ig light chain loci lacking D segments. By contrast, when the VDJ junction on the first IgH allele is nonproductive, the lack of the pre-BCR inhibitory signal allows $V$ to $D$ J recombination on the second allele. If this second attempt is successful, a pre-BCR-mediated proliferation wave will generate abundant $B$ cell clones with biallelic VDJ rearrangements. Roughly half of mature B cells harbor biallelic VDJ-rearrangements with a nonproductivelyrecombined IgH allele (30-32). If another nonproductive VDJ junction occurs on the second IgH allele, cells are eliminated through apoptosis. As mentioned above, pre-BCR signaling stimulates recombination of Ig light chain genes. The presence of two Igא and Ig $\lambda$ light chain isotypes permits multiple VJ recombination events. Again, the expression of a functional BCR precludes further recombination in immature B cells. In humans, $\sim 50 \%$ of mature B cells express $\lg \lambda$. However, in mice, recombination takes places preferentially at the $\lg \kappa_{\kappa}$ locus and only $5 \%$ of B cells express Ig $\lambda$ isotypes (32). Hence, $B$ cells harbor numerous nonproductive $\mathrm{VJ}$-recombined Ig light chain alleles (Fig. 1).

After their exit from the bone marrow, alternative splicing of constant $\mathrm{C} \mu$ and $\mathrm{C} \delta$ exons ensures co-expression of IgM and $\operatorname{lgD}$ at the surface of naïve $B$ cells (33). Upon antigen encounter, IgD expression is downregulated and activated B cells are subjected to a second wave of Ig gene diversification by SHM in germinal centers (GCs). Frequent nonsense mutations can arise during this affinity maturation process that requires transcription of the target region and enzymatic activity of B-cell-specific activation-induced deaminase AID (34). This process leads to the introduction of multiple nucleotide changes in the $\mathrm{V}$ exon (i.e., VDJ or VJ) and a few hundred base pairs in the downstream intron (35). Nucleotide insertions and deletions (indels) have also been observed (36, 37). SHM leads to the expression of a secondary repertoire from which $B$ cells carrying a mutated BCR with improved Ag-binding affinity can be selected (38). We have previously observed that SHM occurs at similar levels on productive and nonproductive VDJ-rearranged IgH alleles (39). If a nonsense codon appears on the productive allele, the lack of Ag-binding activity provokes a rapid elimination of mutated B cell clones within GCs (40-42). The occurrence of SHM on nonproductive Ig alleles can introduce additional nonsense codons, modifying the PTC position within the $\mathrm{V}$ exon. Class switch recombination (CSR) also occurs in germinal centers. This second round of IgH intragenic rearrangements replaces the $\mathrm{C} \mu$ exons with a downstream constant gene (43). GC B cells can differentiate into memory cells or terminally differentiated PCs that secrete substantial amounts of antibody (44). The PC transcriptional program induces major changes including a transcriptional boost of Ig gene transcription and the activation of unfolded protein response (UPR) to ensure proper Ig folding (45). In PCs, the use of secreted polyadenylation signal (PAS) instead of downstream membrane PAS allows alternative $\mathrm{IgH}$ pre-mRNA processing to switch from membrane to secreted Ig isoforms (46). Taken together, the vast majority of B-lineage cells harbor PTC + Ig alleles in their genome with nonsense codons introduced in the $\mathrm{V}$ exon or in the adjacent constant exon during the $\mathrm{V}(\mathrm{D})$ ) recombination process or SHM (Fig. 1).

\section{Transcriptional control of PTC-containing Ig genes}

The high frequency of PTC $+\mathrm{V}(\mathrm{D})$ )-rearranged Ig alleles in $B$-lineage cells needs additional mechanisms to downregulate these nonsense transcripts. A transcriptional silencing of PTC + Ig genes has been proposed as a primary mechanism preventing their expression. This mechanism is called 

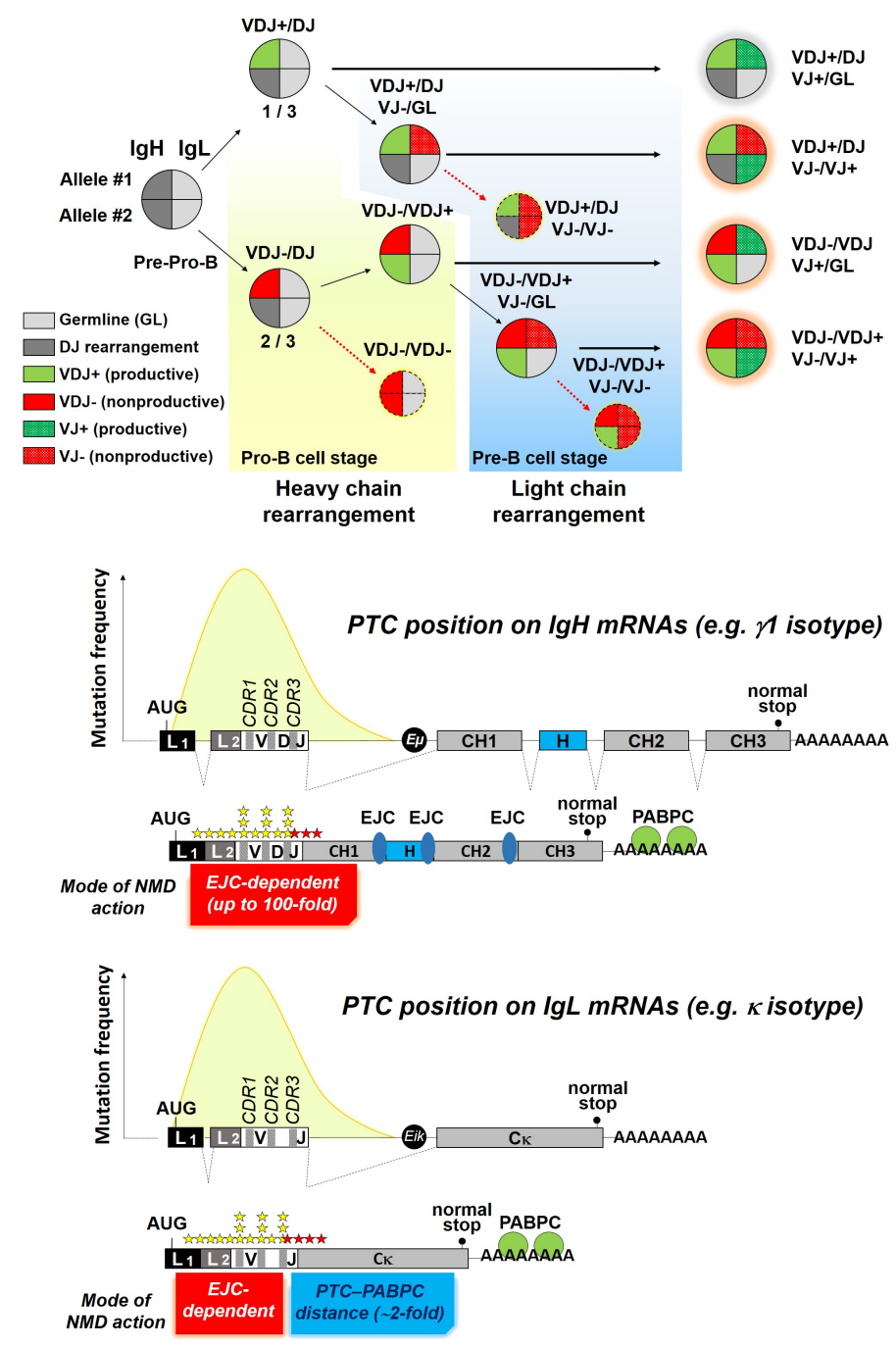

Fig. 1. Abundance of nonproductive $V(D)$ J rearrangements in B-lineage cells. (A) Schematic representation of productive and nonproductive $V(D)$ J rearrangements during the generation of primary antibody repertoire. $V(D)$ ) recombination is initiated by a monoallelic $V$ to $D J$ recombination at the IgH locus (biallelic D-J rearrangements are not depicted). If successful, then $\mathrm{V}$ to J recombination occurs at Ig light chain (IgL) loci. Successive IgL rearrangements are possible due to the fact that there are two Igא and two $\lg \lambda$ alleles (not depicted). Pre-B cell receptor (pre-BCR) or BCR-mediated feedback signalling upon in-frame rearrangement of one $\mathrm{IgH}$ or IgL allele (i.e., $V D J+$ or $\mathrm{VJ}+$ ) prevents $\mathrm{V}(\mathrm{D})$ ) recombination on the second allele (32). By contrast, a nonproductive V(D)J recombination on one Ig allele (i.e. VDJ- or VJ-) induces rearrangement on the second allele. The imprecise nature of $V(D) J$ junctions generates $\sim 1 / 3$ of productive and $\sim 2 / 3$ of nonproductive $V(D)$ J-rearranged alleles. Hence, most $B$-lineage cells harbour nonproductively-recombined Ig alleles in their genome (red parts in pie charts). If the two attempts on both Ig alleles are unsuccessful, the cell is programmed to die by apoptosis (dashed circles). (B) PTCs introduced during the error-prone V(D)J recombination process (red stars) or by somatic hypermutations (SHM; yellow stars) can activate different modes of NMD degradation. Frameshift V(D)J junctions can lead to the appearance of PTCs in the variable (V) exon or in the downstream adjacent constant exon. SHM can lead to the appearance of PTCs in the first leader exon $\left(\mathrm{L}_{1}\right.$ : $\mathrm{L}-$ part1) or in the $\mathrm{V}$ exon, with a greater abundance in the complement-determining regions (CDRs). For lgH mRNAs, PTC introduced by SHM or during V(D)J recombination can elicit exon junction complex (EJC)-dependent NMD. EJCs that remain bound to mRNAs after a pioneer round of translation are depicted (blue ovals). As good NMD candidates, PTC-containing IgH mRNAs are strongly degraded by NMD (up to 100-fold) $(1,9,59)$. However, it has been demonstrated that some nonsense codons in the $5^{\prime}$-half of the VDJ exon could not elicit strong NMD degradation (73). Similarly, PTCs close to the initiation codon are NMD resistant in other models likely due to a critical interaction between PABPC1 with the translation initiation complex $(74,75)$. For nonproductive lgk alleles, PTCs are located at the end of the $\checkmark$ exon or within the last constant Cא exon. Hence, these PTC-containing IgL mRNAs could not elicit EJC-dependent NMD degradation, although they are likely to be targeted by a PTC-PABC1 distance-dependent mode of NMD which induces a less efficient degradation $(\sim 2$-fold) (5). 
"nonsense-mediated transcriptional gene silencing" (NMTGS). It involves chromatin remodelling and "heterochromatinization" of the PTC+ DNA sequence. NMTGS can be inhibited by the overexpression of exonuclease. However, the involvement of siRNA like molecules has not been elucidated yet (4). NMTGS is also impaired upon knock-down of the main NMD factor UPF1, suggesting a mechanistic link between NMD and NMTGS (47). Although NMTGS has been demonstrated in Hela cells transfected with minigene constructs, the occurrence of such a quality control mechanism needs to be determined in B cells. Instead of active silencing, many studies including ours have shown a biallelic transcription pattern for productive and nonproductive Ig alleles in B cells $(9,39,48-51)$. To study the transcription and RNA surveillance of PTC + IgH alleles during $B$ cell development, we introduced a nonsense $V$ exon in the IgH locus to specifically mark each allele in heterozygous mutants. Consistent with previous observations in a pro-B cell line (52), productive and nonproductive IgH alleles exhibited equivalent transcription rates with similar RNAPII loading in LPS-stimulated B cells $(9,48)$. This also confirms our earlier study in germinal center B cells, demonstrating that the frequency of transcription-dependent SHM is similar for productive and nonproductive VDJ-recombined $\mathrm{IgH}$ alleles (39).

\section{RNA SURVEILLANCE IN B-LINEAGE CELLS}

The machinery of RNA surveillance is now known to include various pathways controlling the quality of pre-mRNA and mRNA to limit the translation of truncated proteins. The molecular mechanisms involved in the activation of RNA surveillance pathways have been extensively reviewed previously $(13,53,54)$.

\section{Accumulation of PTC-containing Ig pre-mRNAs}

Despite their active transcription, PTC + Ig genes can be controlled at pre-mRNA level. Previous studies $(2,6)$ performed in Milstein's lab have shown an accumulation of PTC + Igא unspliced or splicing intermediate RNA precursors, supporting the idea that nonsense codons can be recognized in the nucleus by a mechanism independent of protein synthesis. In a simplified model of Igк transcripts containing only three exons, splicing intermediates can eliminate the first intron (IVS1) but retain the intervening sequence between $\mathrm{VJ}$ and $\mathrm{C} \kappa$ exons (IVS2). Upon transfection of B cell lines spanning the main developmental stages with PTC + and PTC-free lgk minigenes, we have observed that the presence of PTC induces a $\sim 2$-fold increase for each unspliced and splicing intermediate RNA precursors (5). Again, unspliced and partially spliced Igא RNAs were mainly confined in the nucleus (5). The accumulation of PTC + precursors was not affected by protein synthesis inhibitors (5). In agreement with these data, RNA-FISH experiments performed in Sp6-derived hybridoma cells further demonstrated that PTC + Ig- $\mu$ pre-mRNAs could accumulate near the site of transcription (55). Recent findings obtained in Drosophila have indicated that UPF1 can bind to nascent RNAs at RNAPII transcription sites to facilitate nuclear processes of gene expression (56). It has been demonstrated that UPF1 is needed for the release of poly(A) mRNAs from chromosomal transcription sites and for their export from the nucleus. It is tempting to speculate that these new nuclear scanning functions of UPF1 could be involved in the nuclear accumulation of nonproductive Ig RNA precursors or in NAS (see below). Interestingly, fluctuations in the steady state level of PTC + IgH pre-mRNAs occurs during B cell development, with low accumulation in PCs compared to resting B cells (9). These observations indicate that a high level of transcription precludes splicing inhibition and accumulation of PTC + Ig pre-mRNAs. Thus, efficient processing of PTC + Ig transcripts in PCs needs additional RNA quality control to prevent the synthesis of truncated Ig polypeptides.

\section{NMD of PTC-containing Ig mRNAs}

NMD is tightly coupled to translation. The molecular mechanisms of the two prevailing exon-junction complex (EJC)-dependent and Poly(A) Binding Protein Cytoplasmic 1 (PTC-PABPC1) distance-dependent NMD activation models have been extensively characterized in the past decades ( 1 , $13,53,54,57,58)$.

According to the "50-55 nucleotides rule" for EJC-dependent NMD activation, the presence of a PTC more than 50-55 nucleotides upstream from the last exon-exon junction can trigger efficient mRNA degradation by NMD. This mode of NMD activation is elicited to downregulate the level of nonproductively-rearranged IgH mRNA that contains PTCs in the $\mathrm{V}$ or in the first constant exon $(\mathrm{CH} 1)$ and several constant exons downstream. Up to 100 -fold accelerated degradation of these NMD candidates has been documented in B cell lines (59-61). Consistent with a previous study using TCR $\beta$ as gene models (62), we found that the extent of NMD was closely correlated to the splicing rate during B-cell development, with $\sim 2$ to 20 -fold degradation of PTC + IgH mRNAs in resting and LPS-stimulated B cells, respectively (9). Interestingly, levels of nonproductive IgH mRNAs remained constantly low in those B cell populations, suggesting compensatory effects between nonsense-mediated splicing inhibition and NMD. Hence, qualitative changes in the RNA surveillance machinery occur during B-cell development. The cooperation between RNA surveillance mechanisms controls the amount of PTC+ IgH mRNAs.

For Igא alleles, the situation is completely different because a frameshift at the VJ junction leads to the appearance of a PTC within the last $\mathrm{C} \kappa$ exon, or less frequently at 3 nucleotides upstream from the last exon-exon junction (50). Thus, the position of PTC on nonproductive Igא mRNAs does not meet the requirements for EJC-dependent NMD. Instead, it belongs to the PTC-PABPC1 distance-dependent NMD activation model. The latter pathway occurs when the 3'UTR of an 
mRNA is abnormally long, as in the case of the presence of a PTC (53). NMD is activated when the $3^{\prime}$ UTR structure precludes the normal interaction of PABPC1 with release factors (eRF1 and eRF3) involved in translation termination. The spatial rearrangement of $3^{\prime} U T R$ controlling the proximity of the termination codon and the poly $(\mathrm{A})$ tail is a critical determinant for NMD (63). Although UPF1 was previously assumed to be the essential player involved in the interaction with release factors (eRFs), in vitro translation termination assays recently performed by Neu-Yilik et al. $(64,65)$ indicate that UPF3B (also named UPF3X) directly interacted with both RNA and eRF3. This NMD mechanism is most likely to be involved in the uptake of nonproductive Igא mRNAs, inducing a modest ( $\sim 2$-fold) NMD degradation in mouse B cells (Fig. 1) (5).

\section{NAS of PTC-containing Ig transcripts and production of deleterious truncated Ig polypeptides in plasma cells}

Mutations within the sequence of exonic splicing enhancers (ESEs) and the presence of a PTC have been shown to elicit alternative splicing called class-I and class-II NAS, respectively $(8,11-13,66,67)$. As an additional RNA surveillance pathway, class-II NAS (hereafter called NAS) can prevent the maturation of full-length PTC-containing mRNAs by promoting alternative splicing to skip the offending PTCs. However, NAS can yield internally deleted mRNAs and proteins. Hence, it exhibits opposite effects compared to NMD with regard to the production of truncated proteins. Although NMD and NAS use the common factor UPF1, these processes are mechanistically different. Knockdown of several key NMD factors including UPF2, UPF3A, UPF3B, and SMG1 showed no significant effect on NAS (13). The intrinsic mechanisms responsible for the activation of NAS remain elusive. Conflicting results have been obtained with regard to the sensitivity of NAS to translation inhibition or frameshift mutations $(5,13)$. Future breakthroughs would indicate whether NAS, nonsense-mediated SOS, or splicing inhibition could be induced as a response to PTC recognition during translation, after nuclear degradation of spliced PTC-containing mRNAs, or by other nuclearscanning mechanisms.

To study NAS of Igא transcripts, we transfected several B-cell lines with minigenes harboring frameshift mutations at the VJ junction that could lead to the appearance of PTCs at the end of the $\mathrm{V}$ exon $\left(\mathrm{V}^{\mathrm{PTC}}\right)$, or within the last $\mathrm{C} \kappa$ exon $\left(\mathrm{C}^{\mathrm{PTC}}\right)(5,16)$. Consistent with a reading frame-dependent NAS activation, the presence of $\mathrm{V}^{\mathrm{PTC}}$, but not of $\mathrm{C}^{\mathrm{PTC}}$, strongly induced skipping of the $V$ exon. We also confirmed that $V$ exon skipping occurred in mouse B cells during splicing of both nonproductive Igא and $\mathrm{IgH}$ transcripts $(5,16,48)$. Interestingly, we found that skipping of the PTC-containing $\mathrm{V}$ exon was greatly increased in PCs compared to B cells. This was correlated with a transcriptional boost of Ig genes during PC differentiation (48). Thus, a fast RNAPII elongation rate can enhance the skipping of PTC-containing V exons in PCs, whereas a slow elongation

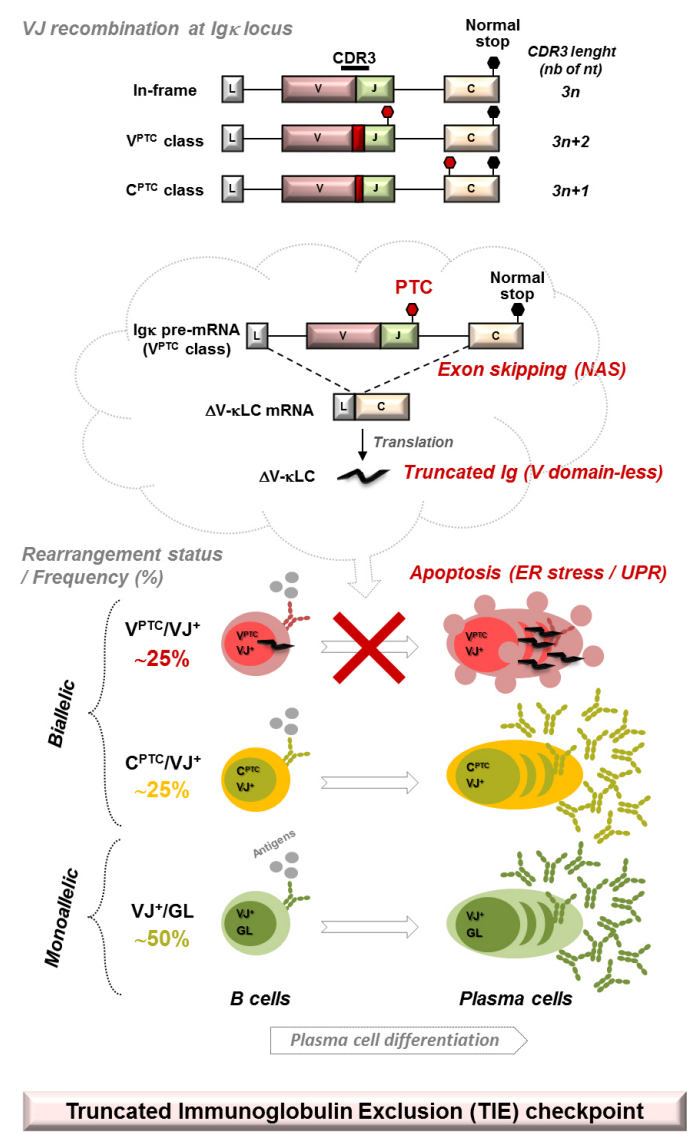

Fig. 2. A Truncated Immunoglobulin Exclusion (TIE) checkpoint during terminal plasma cell differentiation. (Top) Schematic structure of the Igк locus showing various classes of V-J rearrangements. In-frame and out-of-frame VJ junctions with the position of PTCs from the latter are depicted. Any VJ recombination involving mouse $\mathrm{J} \kappa 1$ segments can lead to the appearance of PTCs in the last constant exon (Cא). For other J $\kappa$ segments, the addition of 1 nucleotide (nt) (i.e., $3 \mathrm{n}+1 \mathrm{nt}$ ) at the VJ junction also leads to the appearance of a PTC in the $\mathrm{C} \kappa$ exon, whereas the addition of 2 nt (i.e. $3 n+2 n t)$ creates a PTC at the end of the variable $(\mathrm{V})$ exon, 3nt upstream the exon-exon junction (50). (Middle) Whereas nonsense-associated altered splicing (NAS) is not activated by the presence of a PTC within the $\mathrm{C} \kappa$ exon ( $\mathrm{C}^{\text {PTC }}$ class), PTCs in the $\mathrm{V}$ exon ( $\mathrm{V}^{\text {PTC }}$ class) can strongly promote exon skipping and translation of $\mathrm{V}$ domain-less $\kappa$ light chains $(\Delta \mathrm{V}-\kappa \mathrm{LCs})(5,16)$. (Bottom) The production of $\Delta \mathrm{V}-\kappa \mathrm{LCS}$ is innocuous in $\mathrm{B}$ cells. However, it provokes endoplasmic reticulum (ER) stress-associated apoptosis in plasma cells (PCs), precluding their differentiation as long-lived PCs. Overall, significant numbers of PCs harboring biallelic VJ recombination in a $\mathrm{V}^{\mathrm{PTC}} / \mathrm{NJ}^{+}$configuration at the Igא locus are eliminated through the activation of a Truncated Immunoglobulin Exclusion (TIE) checkpoint (16). The TIE checkpoint exhibits dual effects on antibody responses. On one hand, its activation blunts the PC repertoire that can emerge from mature B cells, thus limiting the extent of antibody responses. On the other hand, the TIE-checkpoint favors the selection of long-lived PCs with limited basal ER stress that supports further elevation of Ig secretion. GL: germline; VJ+: productively- recombined Igא allele; $\mathrm{V}^{\mathrm{PTC}}$ : nonproductively-recombined Igא allele harboring a PTC within the V exon; $C^{\text {PTC }}$ : nonproductively-recombined Igא allele harboring a PTC within the $\mathrm{C} \kappa$ exon. 
rate authorizes their splicing as full-length mRNAs in B cells (or splicing inhibition as mentioned before). These observations are in agreement with the known influence of RNAPII elongation rate on alternative splicing (68), suggesting that PTC-independent and PTC-dependent exon skipping events can be governed by some similar rules.

Until recently, consequences of NAS with regard to the production of truncated Ig chains have been overlooked. Interestingly, we have observed that exon skipping of $\mathrm{V}^{\mathrm{PTC}}$ Igא pre-mRNAs encodes $\mathrm{V}$ domain-less $\kappa$ light chains $(\Delta \mathrm{V}-\kappa \mathrm{LCs})$ that can induce the death of PCs through endoplasmic reticulum (ER) stress-associated apoptosis (16). Revealing a new PC checkpoint referred to as Truncated Immunoglobulin Exclusion (TIE)-checkpoint, the production of $\Delta \mathrm{V}-\kappa \mathrm{LCs}$ can dampen PC differentiation by eliminating cells expressing nonproductively-rearranged $V^{\text {PTC }}$ Igא alleles (Fig. 2). In addition, conditional expression of $\Delta \mathrm{V}-\kappa \mathrm{LC}$ mRNAs in 'inducible-TIE' (iTIE) knock-in mice reproduced physiological TIE checkpoint and affected the survival of long-lived PCs and antibody production (16). Thus, the transcription of nonproductive Ig alleles is not as safe as previously assumed and the activation of NAS can drive the elimination of PC clones harbouring biallelic Igא rearrangements. Remarkably, the TIE-checkpoint can reduce the magnitude of humoral responses and shape the antibody repertoire independently of classical constraints related to Ag specificity.

\section{Closing remarks}

Aberrantly rearranged Ig alleles are abundant in B-lineage cells. The cooperative action of nonsense-mediated splicing inhibition (or SOS) and NMD limits the amount of deleterious truncated Ig polypeptides. However, activation of the NAS pathway exerts opposite effects with exon skipping-mediated production of $\mathrm{V}$ domain-less Ig chains. In PCs with biallelic Igא rearrangements, transcriptional boost of Ig genes can sustain massive Ig synthesis. However, it also promotes NAS activation and exon skipping during splicing of nonproductive transcripts. Because the survival of normal and malignant PCs is tightly controlled by ER stress and proteasome activity (69), high amounts of truncated Ig can provoke PC death as a consequence of a deregulated proteostasis. According to an efficient TIE-checkpoint, alternatively spliced mRNAs encoding structurally abnormal Ig chains are hardly detectable in non-malignant PCs, although they can be observed in some lymphoproliferative disorders, including Multiple Myeloma and Burkitt lymphoma (70-72). Previous findings have indicated that the activation of the TIE checkpoint can eliminate $\sim 20-25 \%$ of PCs, i.e., those expressing nonproductive $\mathrm{V}^{\mathrm{PTC}}$ Igא alleles (16). If a similar disappearance of PC harboring nonproductive VDJ rearrangements is observed for the $\mathrm{IgH}$ repertoire, it could indicate a broad TIE-checkpoint blunting the terminal differentiation of many PCs with biallelic IgH and/or IgL rearrangements. Altogether, this should have major consequences in our classical mindset usually considering nonproductively $\mathrm{V}(\mathrm{D})$ )-rearranged $\mathrm{Ig}$ alleles as passengers rather than drivers.

\section{ACKNOWLEDGEMENTS}

This work was supported by grants from Fondation ARC (PJA 20161204724), INCa (PLBIO15-256), ANR (2017-CE15-0024-01), Ligue Contre le Cancer (CD87, CD19, CD23), and Fondation Française pour la Recherche contre le Myélome et les Gammapathies monoclonales (FFRMG). JML was funded by French government and Ligue Contre le Cancer fellowships. NS was funded by Région Limousin and Fondation ARC fellowships.

\section{CONFLICTS OF INTEREST}

The authors have no conflicting interests.

\section{REFERENCES}

1. Li S and Wilkinson MF (1998) Nonsense surveillance in lymphocytes? Immunity 8, 135-141

2. Aoufouchi S, Yélamos J and Milstein C (1996) Nonsense mutations inhibit RNA splicing in a cell-free system: recognition of mutant codon is independent of protein synthesis. Cell 85, 415-422

3. Bühler M and Mühlemann O (2005) Alternative splicing induced by nonsense mutations in the immunoglobulin mu VDJ exon is independent of truncation of the open reading frame. RNA 11, 139-146

4. Bühler M, Mohn F, Stalder L and Mühlemann O (2005) Transcriptional silencing of nonsense codon-containing immunoglobulin minigenes. Mol Cell 18, 307-317

5. Chemin G, Tinguely A, Sirac C et al (2010) Multiple RNA surveillance mechanisms cooperate to reduce the amount of nonfunctional Ig kappa transcripts. J Immunol Baltim Md 1950 184, 5009-5017

6. Lozano F, Maertzdorf B, Pannell R and Milstein C (1994) Low cytoplasmic mRNA levels of immunoglobulin kappa light chain genes containing nonsense codons correlate with inefficient splicing. EMBO J 13, 4617-4622

7. Mendell JT, ap Rhys CM and Dietz HC (2002) Separable roles for rent $1 / \mathrm{hUpf} 1$ in altered splicing and decay of nonsense transcripts. Science 298, 419-422

8. Sperling J and Sperling R (2008) Nuclear surveillance of RNA polymerase II transcripts. RNA Biol 5, 220-224

9. Tinguely A, Chemin G, Péron $S$ et al (2012) Cross talk between immunoglobulin heavy-chain transcription and RNA surveillance during B cell development. Mol Cell Biol 32, 107-117

10. Wachtel C, Li B, Sperling J and Sperling R (2004) Stop codon-mediated suppression of splicing is a novel nuclear scanning mechanism not affected by elements of protein synthesis and NMD. RNA 10, 1740-1750

11. Wang J, Hamilton JI, Carter MS, Li S and Wilkinson MF (2002) Alternatively spliced TCR mRNA induced by disruption of reading frame. Science 297, 108-110 
12. Wang J, Chang YF, Hamilton JI and Wilkinson MF (2002) Nonsense-associated altered splicing: a frame-dependent response distinct from nonsense-mediated decay. Mol Cell 10, 951-957

13. Hwang J and Kim YK (2013) When a ribosome encounters a premature termination codon. BMB Rep 46, 9-16

14. Frischmeyer-Guerrerio PA, Montgomery RA, Warren DS et al (2011) Perturbation of thymocyte development in nonsense-mediated decay (NMD)-deficient mice. Proc Natl Acad Sci U S A 108, 10638-10643

15. Weischenfeldt J, Damgaard I, Bryder D et al (2008) NMD is essential for hematopoietic stem and progenitor cells and for eliminating by-products of programmed DNA rearrangements. Genes Dev 22, 1381-1396

16. Srour N, Chemin G, Tinguely A et al (2016) A plasma cell differentiation quality control ablates B cell clones with biallelic Ig rearrangements and truncated Ig production. J Exp Med 213, 109-122

17. Jung D, Giallourakis C, Mostoslavsky R and Alt FW (2006) Mechanism and control of $\mathrm{V}(\mathrm{D})$ ) recombination at the immunoglobulin heavy chain locus. Annu Rev Immunol 24, 541-570

18. Mombaerts $\mathrm{P}$, lacomini J, Johnson RS, Herrup K, Tonegawa $S$ and Papaioannou VE (1992) RAG-1-deficient mice have no mature B and T lymphocytes. Cell 68, 869-877

19. Shinkai Y, Rathbun G, Lam KP et al (1992) RAG-2-deficient mice lack mature lymphocytes owing to inability to initiate V(D)J rearrangement. Cell 68, 855-867

20. Alt FW and Baltimore D (1982) Joining of immunoglobulin heavy chain gene segments: implications from a chromosome with evidence of three D-JH fusions. Proc Natl Acad Sci U S A 79, 4118-4122

21. Roth DB, Chang XB and Wilson JH (1989) Comparison of filler DNA at immune, nonimmune, and oncogenic rearrangements suggests multiple mechanisms of formation. Mol Cell Biol 9, 3049-3057

22. Desiderio SV, Yancopoulos GD, Paskind M et al (1984) Insertion of $\mathrm{N}$ regions into heavy-chain genes is correlated with expression of terminal deoxytransferase in B cells. Nature 311, 752-755

23. Gilfillan S, Dierich A, Lemeur M, Benoist $C$ and Mathis D (1993) Mice lacking TdT: mature animals with an immature lymphocyte repertoire. Science 261, 1175-1178

24. Kallenbach S, Doyen N, Fanton d'Andon M and Rougeon F (1992) Three lymphoid-specific factors account for all junctional diversity characteristic of somatic assembly of T-cell receptor and immunoglobulin genes. Proc Natl Acad Sci U S A 89, 2799-2803

25. Komori T, Okada A, Stewart V and Alt FW (1993) Lack of $\mathrm{N}$ regions in antigen receptor variable region genes of TdT-deficient lymphocytes. Science 261, 1171-1175

26. Lafaille JJ, DeCloux A, Bonneville M, Takagaki Y and Tonegawa S (1989) Junctional sequences of T cell receptor gamma delta genes: implications for gamma delta $T$ cell lineages and for a novel intermediate of $\mathrm{V}$-(D)-J joining. Cell 59, 859-870

27. Lewis SM (1994) $P$ nucleotide insertions and the resolution of hairpin DNA structures in mammalian cells. Proc Natl Acad Sci U S A 91, 1332-1336

28. McCormack WT, Tjoelker LW, Carlson LM et al (1989)
Chicken IgL gene rearrangement involves deletion of a circular episome and addition of single nonrandom nucleotides to both coding segments. Cell 56, 785-791

29. Outters $P$, Jaeger $S$, Zaarour $N$ and Ferrier $P$ (2015) Long-Range Control of V(D)J Recombination \& Allelic Exclusion: Modeling Views. Adv Immunol 128, 363-413

30. ten Boekel E, Melchers F and Rolink A (1995) The status of Ig loci rearrangements in single cells from different stages of B cell development. Int Immunol 7, 1013-1019

31. Ehlich A, Martin V, Müller W and Rajewsky K (1994) Analysis of the B-cell progenitor compartment at the level of single cells. Curr Biol CB 4, 573-583

32. Mostoslavsky R, Alt FW and Rajewsky K (2004) The lingering enigma of the allelic exclusion mechanism. Cell $118,539-544$

33. Gutzeit C, Chen K and Cerutti A (2018) The enigmatic function of IgD: some answers at last. Eur J Immunol 48, 1101-1113

34. Honjo T, Nagaoka $H$, Shinkura $R$ and Muramatsu $M$ (2005) AID to overcome the limitations of genomic information. Nat Immunol 6, 655-661

35. Pilzecker B and Jacobs H (2019) Mutating for Good: DNA Damage Responses During Somatic Hypermutation. Front Immunol 10, 438

36. Goossens T, Klein U and Küppers R (1998) Frequent occurrence of deletions and duplications during somatic hypermutation: Implications for oncogene translocations and heavy chain disease. Proc Natl Acad Sci U S A 95, 2463-2468

37. Yeap LS, Hwang JK, Du Z et al (2015) Sequence-Intrinsic Mechanisms that Target AID Mutational Outcomes on Antibody Genes. Cell 163, 1124-1137

38. Manser T, Tumas-Brundage KM, Casson LP et al (1998) The roles of antibody variable region hypermutation and selection in the development of the memory B-cell compartment. Immunol Rev 162, 183-196

39. Delpy L, Sirac C, Le Morvan C and Cogné M (2004) Transcription-dependent somatic hypermutation occurs at similar levels on functional and nonfunctional rearranged IgH alleles. J Immunol Baltim Md 1950 173, 1842-1848

40. Allen CD, Okada T and Cyster JG (2007) Germinal-center organization and cellular dynamics. Immunity 27, 190-202

41. Stewart I, Radtke D, Phillips B, McGowan SJ and Bannard O (2018) Germinal Center B Cells Replace Their Antigen Receptors in Dark Zones and Fail Light Zone Entry when Immunoglobulin Gene Mutations are Damaging. Immunity 49, 477-489.e7

42. Victora GD and Nussenzweig MC (2012) Germinal centers. Annu Rev Immunol 30, 429-457

43. Manis JP, Tian M and Alt FW (2002) Mechanism and control of class-switch recombination. Trends Immunol 23, 31-39

44. Nutt SL, Hodgkin PD, Tarlinton DM and Corcoran LM (2015) The generation of antibody-secreting plasma cells. Nat Rev Immunol 15, 160-171

45. Todd DJ, McHeyzer-Williams LJ, Kowal C et al (2009) XBP1 governs late events in plasma cell differentiation and is not required for antigen-specific memory B cell development. J Exp Med 206, 2151-2159

46. Takagaki Y, Seipelt RL, Peterson ML and Manley JL (1996) 
The polyadenylation factor CstF-64 regulates alternative processing of IgM heavy chain pre-mRNA during B cell differentiation. Cell 87, 941-952

47. Stalder L and Mühlemann O (2007) Transcriptional silencing of nonsense codon-containing immunoglobulin micro genes requires translation of its mRNA. J Biol Chem 282, 16079-16085

48. Ashi MO, Srour N, Lambert JM et al (2018) Physiological and druggable skipping of immunoglobulin variable exons in plasma cells. Cell Mol Immunol 16, 810-819

49. Daly J, Licence S, Nanou A, Morgan G and Mårtensson IL (2007) Transcription of productive and nonproductive VDJ-recombined alleles after IgH allelic exclusion. EMBO J 26, 4273-4282

50. Delpy L, Sirac C, Magnoux E, Duchez $S$ and Cogné $M$ (2004) RNA surveillance down-regulates expression of nonfunctional kappa alleles and detects premature termination within the last kappa exon. Proc Natl Acad Sci U S A 101, 7375-7380

51. Holwerda SJ, van de Werken HJ, Ribeiro de Almeida C et al (2013) Allelic exclusion of the immunoglobulin heavy chain locus is independent of its nuclear localization in mature B cells. Nucleic Acids Res 41, 6905-6916

52. Eberle $A B$, Herrmann $K$, Jäck $H M$ and Mühlemann $O$ (2009) Equal transcription rates of productively and nonproductively rearranged immunoglobulin mu heavy chain alleles in a pro-B cell line. RNA 15, 1021-1028

53. Lejeune $F$ (2017) Nonsense-mediated mRNA decay at the crossroads of many cellular pathways. BMB Rep 50, 175-185

54. Lejeune F and Maquat LE (2005) Mechanistic links between nonsense-mediated mRNA decay and pre-mRNA splicing in mammalian cells. Curr Opin Cell Biol 17, 309-315

55. Mühlemann O, Mock-Casagrande CS, Wang J et al (2001) Precursor RNAs harboring nonsense codons accumulate near the site of transcription. Mol Cell 8, 33-43

56. Singh AK, Choudhury SR, De $S$ et al (2019) The RNA helicase UPF1 associates with mRNAs co-transcriptionally and is required for the release of mRNAs from gene loci. ELife 8, pii: e41444

57. Chang YF, Imam JS and Wilkinson MF (2007) The nonsense-mediated decay RNA surveillance pathway. Annu Rev Biochem 76, 51-74

58. Karousis ED and Mühlemann O (2019) Nonsense-Mediated mRNA Decay Begins Where Translation Ends. Cold Spring Harb Perspect Biol 11, pii: a032862

59. Baumann B, Potash MJ and Köhler G (1985) Consequences of frameshift mutations at the immunoglobulin heavy chain locus of the mouse. EMBO J 4, 351-359

60. Connor A, Wiersma E and Shulman MJ (1994) On the linkage between RNA processing and RNA translatability.
J Biol Chem 269, 25178-25184

61. Jäck HM, Berg J and Wabl M (1989) Translation affects immunoglobulin mRNA stability. Eur J Immunol 19, 843-847

62. Gudikote JP, Imam JS, Garcia RF and Wilkinson MF (2005) RNA splicing promotes translation and RNA surveillance. Nat Struct Mol Biol 12, 801-809

63. Eberle $A B$, Stalder $L$, Mathys $H$, Orozco RZ and Mühlemann O (2008) Posttranscriptional Gene Regulation by Spatial Rearrangement of the 3' Untranslated Region. PLoS Biol 6, e92

64. Kim YK and Maquat LE (2019) UPFront and center in RNA decay: UPF1 in nonsense-mediated mRNA decay and beyond. RNA N Y N 25, 407-422

65. Neu-Yilik G, Raimondeau E, Eliseev B et al (2017) Dual function of UPF3B in early and late translation termination. EMBO J 36, 2968-2986

66. Maquat LE (2002) NASty effects on fibrillin pre-mRNA splicing: another case of ESE does it, but proposals for translation-dependent splice site choice live on. Genes Dev 16, 1743-1753

67. Valentine CR (1998) The association of nonsense codons with exon skipping. Mutat Res 411, 87-117

68. Nogués G, Kadener S, Cramer P et al (2003) Control of alternative pre-mRNA splicing by RNA Pol II elongation: faster is not always better. IUBMB Life 55, 235-241

69. Cenci $S$ and Sitia R (2007) Managing and exploiting stress in the antibody factory. FEBS Lett 581, 3652-3657

70. Cogné $M$ and Guglielmi $P$ (1993) Exon skipping without splice site mutation accounting for abnormal immunoglobulin chains in nonsecretory human myeloma. Eur J Immunol 23, 1289-1293

71. Cogné $M$, Bakhshi $A$, Korsmeyer SJ and Guglielmi $P$ (1988) Gene mutations and alternate RNA splicing result in truncated Ig L chains in human gamma $\mathrm{H}$ chain disease. J Immunol Baltim Md 1950 141, 1738-1744

72. Cogné $M$, Silvain $C$, Khamlichi AA and Preud'homme JL (1992) Structurally abnormal immunoglobulins in human immunoproliferative disorders. Blood 79, 2181-2195

73. Bühler M, Paillusson A and Mühlemann O (2004) Efficient downregulation of immunoglobulin mu mRNA with premature translation-termination codons requires the 5 '-half of the VDJ exon. Nucleic Acids Res 32, 3304-3315

74. Inácio A, Silva AL, Pinto J et al (2004) Nonsense mutations in close proximity to the initiation codon fail to trigger full nonsense-mediated mRNA decay. J Biol Chem 279, 32170-32180

75. Peixeiro I, Inácio $\hat{A}$, Barbosa C, Silva AL, Liebhaber SA and Romão L (2012) Interaction of PABPC1 with the translation initiation complex is critical to the NMD resistance of AUG-proximal nonsense mutations. Nucleic Acids Res 40, 1160-1173 